\title{
Experimental Comparison of Efficient Tomography Schemes for a Six-Qubit State
}

\author{
Christian Schwemmer, ${ }^{1,2}$ Géza Tóth, ${ }^{3,4,5}$ Alexander Niggebaum, ${ }^{6}$ \\ Tobias Moroder, ${ }^{7}$ David Gross, ${ }^{8}$ Otfried Gühne, ${ }^{7}$ and Harald Weinfurter ${ }^{1,2}$ \\ ${ }^{1}$ Max-Planck-Institut für Quantenoptik, Hans-Kopfermann-Straße 1, D-85748 Garching, Germany \\ ${ }^{2}$ Department für Physik, Ludwig-Maximilians-Universität, D-80797 München, Germany \\ ${ }^{3}$ Department of Theoretical Physics, University of the Basque Country UPV/EHU, P.O. Box 644, E-48080 Bilbao, Spain \\ ${ }^{4}$ IKERBASQUE, Basque Foundation for Science, E-48011 Bilbao, Spain \\ ${ }^{5}$ Wigner Research Centre for Physics, Hungarian Academy of Sciences, P.O. Box 49, H-1525 Budapest, Hungary \\ ${ }^{6}$ School of Physics and Astronomy, University of Birmingham, B15 2TT Birmingham, United Kingdom \\ ${ }^{7}$ Naturwissenschaftlich-Technische Fakultät, Universität Siegen, Walter-Flex-Straße 3, D-57068 Siegen, Germany \\ ${ }^{8}$ Physikalisches Institut \& FDM, Universität Freiburg, Rheinstraße 10, D-79104 Freiburg, Germany
}

(Received 29 January 2014; published 24 July 2014)

\begin{abstract}
Quantum state tomography suffers from the measurement effort increasing exponentially with the number of qubits. Here, we demonstrate permutationally invariant tomography for which, contrary to conventional tomography, all resources scale polynomially with the number of qubits both in terms of the measurement effort as well as the computational power needed to process and store the recorded data. We demonstrate the benefits of combining permutationally invariant tomography with compressed sensing by studying the influence of the pump power on the noise present in a six-qubit symmetric Dicke state, a case where full tomography is possible only for very high pump powers.
\end{abstract}

DOI: 10.1103/PhysRevLett.113.040503

PACS numbers: 03.67.Mn, 03.65.Wj, 42.50.Dv

Introduction.-The number of controllable qubits in quantum experiments is steadily growing [1,2]. Yet, to fully characterize a multiqubit state via quantum state tomography (QST), the measurement effort scales exponentially with the number of qubits. Moreover, the amount of data to be saved and the resources to process them scale exponentially, too. Thus, the limit of conventional QST will soon be reached. The following question arises: how much information about a quantum state can be inferred without all the measurements a full QST would require? Protocols have been proposed which need significantly fewer measurement settings if one has additional knowledge about a state, e.g., that it is of low rank, a matrix product state, or a permutationally invariant (PI) state [3-8]. Some of these approaches only require a polynomially increasing number of measurements and even offer scalable postprocessing algorithms [5,8]. Yet, it is important to test the different approaches and evaluate their results for various quantum states.

Here, we implement and compare four different QST schemes in a six-photon experiment. In detail, we perform the largest QST of a photonic multiqubit state so far. We use these data as a reference for a detailed evaluation of different tomography schemes, which enable the state determination with significantly fewer measurements. The recently proposed, scalable PI analysis is implemented

Published by the American Physical Society under the terms of the Creative Commons Attribution 3.0 License. Further distribution of this work must maintain attribution to the author(s) and the published article's title, journal citation, and DOI. here and thus enables us, for the first time, to also perform the numerical evaluation with polynomial resources only. We evaluate the convergence of compressed sensing (CS) schemes and show that the combination of PI and CS can further reduce the measurement effort, without sacrificing performance. We demonstrate the usability of these significantly improved methods to characterize the effects of higher-order emission in spontaneous parametric downconversion (SPDC), an analysis which would not have been possible without the novel tomography schemes.

Scalable scheme for measurements. - Let us first consider the measurement effort needed for tomography. For full QST, each $N$-qubit state is associated with a normalized non-negative Hermitian matrix $\varrho$ with $4^{N}-1$ real free parameters. Since all free parameters have to be determined, any scheme that is suitable to fully analyzing an arbitrary state, such as, e.g., the standard Pauli tomography scheme, suffers from an exponentially increasing measurement effort $[9,10]$. PI states, in contrast, are described by only $\left(\begin{array}{c}N+3 \\ N\end{array}\right)-1=O\left(N^{3}\right)$ free parameters. Tomography in the PI subspace can be performed by measuring (global) operators of the form $A_{i}^{\otimes N}$ with $A_{i}=\vec{n}_{i} \vec{\sigma}$, i.e., measurements of the polarization along the same direction $\vec{n}_{i}$ for every photon [7]. Here, $\left|\vec{n}_{i}\right|=1$ and $\vec{\sigma}=\left(\sigma_{x}, \sigma_{y}, \sigma_{z}\right)$ with Pauli operators $\sigma_{i}(i=x, y, z)$. Each single measurement setting $A_{i}^{\otimes N}$ delivers $N$ expectation values of the operators $M_{i}^{n}=(1 / N !) \sum_{k} \Pi_{k}\left[|0\rangle_{i}\left\langle\left. 0\right|^{\otimes(N-n)} \otimes \mid 1\right\rangle_{i}\left\langle\left. 1\right|^{\otimes n}\right] \Pi_{k}^{\dagger}\right.$, where the summation is over all permutations $\Pi_{k}$ and $i$ refers to the eigenbasis of $A_{i}$. This reduces the number of necessary 
settings to $\mathcal{D}_{N}=\left(\begin{array}{c}N+2 \\ N\end{array}\right)=\frac{1}{2}\left(N^{2}+3 N+2\right)=O\left(N^{2}\right)$. Note that if one allows global entangled measurements, this number can be further reduced [11]. Most importantly, whether an unknown $N$-qubit state is close to being PI can be checked in advance by measuring the settings $\sigma_{x}^{\otimes N}, \sigma_{y}^{\otimes N}$, and $\sigma_{z}^{\otimes N}$. These measurements are already sufficient to give a lower bound for the overlap with the symmetric subspace [7,12].

Scalable representation of states and operators.-The above approach not only reduces the experimental effort, it also offers the possibility to efficiently store and process the measured data. Describing states in the PI subspace enables an efficient representation with only polynomial scaling of the storage space and processing time $[8,13]$.

Consider the angular momentum basis states $\left|j, j_{z}, \alpha\right\rangle$ for the $N$-qubit Hilbert space, with $\vec{J}^{2}\left|j, j_{z}, \alpha\right\rangle=$ $j(j+1)\left|j, j_{z}, \alpha\right\rangle$ and $J_{z}\left|j, j_{z}, \alpha\right\rangle=j_{z}\left|j, j_{z}, \alpha\right\rangle$, where the total spin numbers are restricted to be $j=j_{\min }, j_{\min }+$ $1, \ldots, N / 2$ starting from $j_{\min }=0$ for $N$ even and $j_{\min }=\frac{1}{2}$ for $N$ odd, while $j_{z}=-(N / 2),-(N / 2)+1, \ldots, N / 2$. Here, $\alpha=1,2, \ldots, d_{j}$ is a label to remove the degeneracy (of degree $d_{j}$ [14]) of the eigenstates of $\vec{J}^{2}$ and $J_{z}^{2}$. In this basis, PI states can be written in a simple block diagonal form

$$
Q_{\mathrm{PI}}=\bigoplus_{j=j_{\min }}^{N / 2} \frac{\mathbb{1}_{d_{j}}}{d_{j}} \otimes p_{j} Q_{j}
$$

with $Q_{j}$ being the density operators of the spin- $j$ subspace and $p_{j}$ a probability distribution. Hence, it is sufficient to consider only the $N / 2$ blocks $\tilde{\varrho}_{j}=p_{j} \varrho_{j} / d_{j}$ (of which each has a multiplicity of $d_{j}$; see Fig. 1) with the largest block - the symmetric subspace-being of dimension $(N+1) \times(N+1)$ and multiplicity $d_{N / 2}=1$. Consequently, a PI state can be stored efficiently.

Even if the state to be analyzed is not PI, as long as the observable to be measured is PI, one can hugely benefit from the scheme, since a similarly scalable decomposition can be found for any PI operator $O$, i.e., $O=\bigoplus_{j} \mathbb{1}_{d_{j}} \otimes O_{j}$. Together with Eq. (1), this yields an efficient way to also calculate the expectation values $\langle O\rangle=\operatorname{Tr}(\varrho O)=$

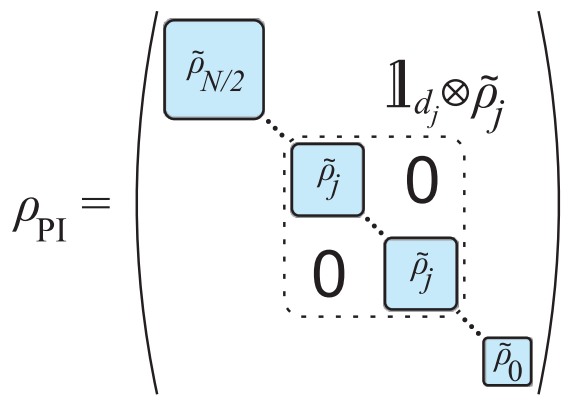

FIG. 1 (color online). Every PI state can be decomposed into a block diagonal form. Exemplarily shown is the combination of $d_{j}$ block matrices $\tilde{\varrho_{j}}$ which are all identical. $\sum_{j} p_{j} \operatorname{Tr}\left(\varrho_{j} O_{j}\right)$ for non-PI states. Note that while, in the regular case, the trace has to be taken over the product of two $2^{N}$-dimensional matrices, now we only have about $\frac{N}{2}$ terms with traces of at most $(N+1)$-dimensional matrices. Again, the effort reduces from exponential to polynomial. For the six-qubit case $\left(j \in j_{\min }=0,1,2, N / 2=3\right)$, this means that the state to be analyzed as well as each measurement operator can be described by only four Hermitian matrices of size $7 \times 7,5 \times 5,3 \times 3$, and $1 \times 1$, respectively, reducing the number of parameters from $4^{6}-1=4095$ to $\left(\begin{array}{l}9 \\ 6\end{array}\right)-1=83$ only.

Data analysis starts with the counts $c_{i}^{n}$ observed measuring $M_{i}^{n}$ and the frequencies $f_{i}^{n}=c_{i}^{n} / \sum_{k} c_{i}^{k}$, respectively. Solving the system of linear equations $f_{i}^{n} \approx\left\langle M_{i}^{n}\right\rangle=$ $\operatorname{Tr}\left(\varrho M_{i}^{n}\right)$ for the free parameters of $\varrho$ usually results in a nonpositive and thus unphysical density matrix $(\varrho \geq 0)$ due to statistical errors and misalignment. Here, typically, a maximum likelihood (ML) fitting algorithm is used to find the physical state that optimally agrees with the measured data $[9,15,16]$. We use convex optimization $[8,19]$, which guarantees a unique minimum and fast convergence. The performance of our algorithm is illustrated best by the fact that a 20-qubit PI state can be reconstructed in fewer than 10 min on a standard desktop computer.

State reconstruction of low rank states and compressed sensing.-As shown recently, low rank states, i.e., states with only a few nonzero eigenvalues, enable state reconstruction even if the underlying set of data obtained from random Pauli measurements is incomplete [3]. There, the measurement effort to analyze a state of rank $r$ with $r 2^{N}$ free parameters scales like $O\left(r 2^{N} \log 2^{N}\right)$ —clearly achieving optimal scaling up to a $\log$ factor. Despite the still exponential scaling, the square root improvement can be considerable. Since, in many cases, the state to be experimentally prepared is at the same time PI and of low rank, we demonstrate here for the first time that combining the two methods is possible [16,20].

Experimental state tomography.-Let us now compare the various QST schemes. In particular, we evaluate the number of settings necessary to obtain (almost) full knowledge about the state. As a reference, we perform, for the first time, full QST of a six-photon state. This is possible only at very high pump power $(8.4 \mathrm{~W})$ of the downconversion source where we collect data for the complete set of Pauli settings. PI tomography is performed to test it against full QST and to analyze states emitted for lower pump powers. For both strategies, we also analyze the convergence of CS tomography for incomplete data.

The six-photon state observed in this work is the symmetric Dicke state $\left|D_{6}^{(3)}\right\rangle$. In general, symmetric Dicke states are defined as

$$
\left|D_{N}^{(n)}\right\rangle=\left(\begin{array}{c}
N \\
n
\end{array}\right)^{-1 / 2} \sum_{i} \mathcal{P}_{i}\left(\left|H^{\otimes(N-n)}\right\rangle \otimes\left|V^{\otimes n}\right\rangle\right),
$$


TABLE I. Overlap with the symmetric Dicke states determined from full tomography, PI tomography with 28 settings, CS with 270 settings, and CS in the PI subspace (PI,CS) with 16 settings. The fidelities for all tomography schemes were determined from the respective ML reconstructed states. Nonparametric bootstrapping [23] was performed from which the corresponding standard deviations were determined as $<0.005,<0.015$, $<0.008$, and $<0.020$ for full tomography, PI tomography, $\mathrm{CS}$, and CS in the PI subspace, respectively.

\begin{tabular}{lcccc}
\hline \hline State & Full & PI & CS & PI, CS \\
\hline$\left|D_{6}^{(0)}\right\rangle$ & 0.001 & 0.001 & 0.001 & 0.002 \\
$\left|D_{6}^{(1)}\right\rangle$ & 0.005 & 0.008 & 0.011 & 0.006 \\
$\left|D_{6}^{(2)}\right\rangle$ & 0.197 & 0.222 & 0.181 & 0.207 \\
$\left|D_{6}^{(3)}\right\rangle$ & 0.604 & 0.590 & 0.615 & 0.592 \\
$\left|D_{6}^{(4)}\right\rangle$ & 0.122 & 0.127 & 0.118 & 0.119 \\
$\left|D_{6}^{(5)}\right\rangle$ & 0.003 & 0.004 & 0.003 & 0.005 \\
$\left|D_{6}^{(6)}\right\rangle$ & 0.000 & 0.003 & 0.001 & 0.004 \\
$\sum$ & 0.933 & 0.954 & 0.929 & 0.935 \\
\hline \hline
\end{tabular}

where $|H / V\rangle_{i}$ denotes horizontal or vertical polarization in the $i$ th mode and the $\mathcal{P}_{i}$ represent all the distinct permutations. In order to experimentally observe $\left|D_{6}^{(3)}\right\rangle$, we distribute an equal number of $H$ and $V$ polarized photons over six output modes and apply conditional detection (for details, see the Supplemental Material [16] and Ref. [21]). The setup uses cavity enhanced SPDC [22] with special care taken to further reduce losses of all components and to optimize the yield of $\left|D_{6}^{(3)}\right\rangle$.

Data are recorded at a pump power of $8.40 \pm 0.56 \mathrm{~W}$ over 4 min for each of the $3^{6}=729$ Pauli settings. The sixphoton count rate was 58 events per minute on average, leading to about 230 events per basis setting within a total measurement time of approximately $50 \mathrm{~h} \mathrm{[24].} \mathrm{The}$ reconstructed density matrix can be seen in Fig. 2(a). Table I lists the fidelity [25] with all the various Dicke states. Their sum reaches high values, proving that the state is close to the symmetric subspace.

Evidently, the experimental state is a mixture of mainly $\left|D_{6}^{(2)}\right\rangle,\left|D_{6}^{(3)}\right\rangle$, and $\left|D_{6}^{(4)}\right\rangle$, and thus CS might be used beneficially. The following question arises: how many settings are required for CS for a faithful reconstruction of the state? We chose random subsets of up to 300 settings from the 729 settings for full tomography. Figure 2(d) gives the probability distribution of the fidelity of the reconstructed matrix for a bin size of 0.01 with respect to the results of full tomography. While, for a low number of settings $(<10)$, the results are randomly spread out, the overlap is already, on average, $\geq 0.800$ for 20 settings. We find that to reach a fidelity of $\geq 0.950$, one requires about 270 settings. Figure $2(\mathrm{c})$ shows the density matrix obtained from 270 settings $\left[F\left(\varrho_{\mathrm{CS}}, \varrho_{\text {full }}\right)=0.950\right]$.

PI tomography should be clearly more efficient. To test its applicability, we first determined the lower bound for the
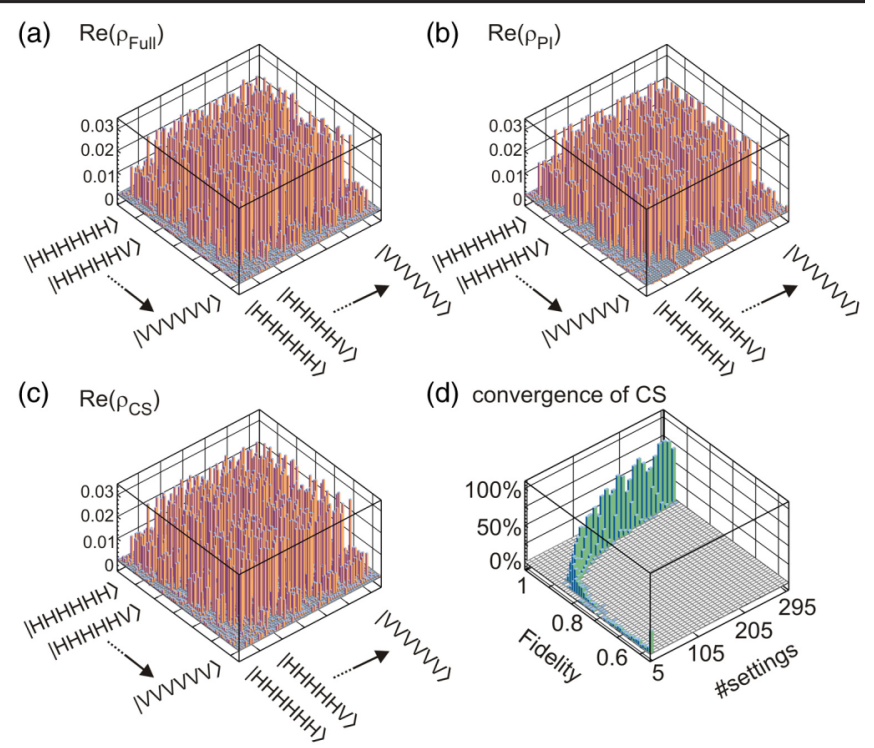

(b) $\operatorname{Re}\left(\rho_{\mathrm{Pl}}\right)$

(d) convergence of CS

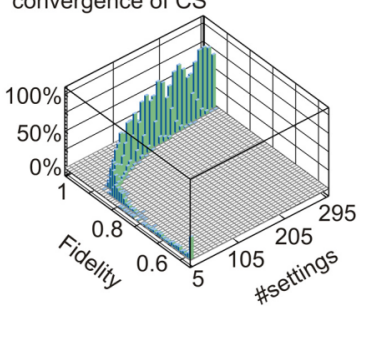

FIG. 2 (color online). ML reconstruction of the state $\left|D_{6}^{(3)}\right\rangle$ obtained from (a) full and (b) PI tomography and (c) CS with 270 settings performed at a pump power of $8.4 \mathrm{~W}$. The respective fidelities are $0.604,0.590$, and 0.615 with mutual overlaps of $F\left(\varrho_{\text {full }}, \varrho_{\mathrm{PI}}\right)=0.922, F\left(\varrho_{\text {full }}, \varrho_{\mathrm{CS}}\right)=0.950$, and $F\left(\varrho_{\mathrm{PI}}, \varrho_{\mathrm{CS}}\right)=$ 0.908. (d) Probability to obtain a certain fidelity for CS with a certain number of randomly chosen settings in comparison with full tomography.

projection of the state onto the symmetric subspace, i.e., the largest block in Fig. $1,\left\langle P_{s}^{(6)}\right\rangle$ from the settings $\sigma_{x}^{\otimes 6}, \sigma_{y}^{\otimes 6}$, and $\sigma_{z}^{\otimes 6}$ by analyzing all photons under $\pm 45^{\circ}$, right- or left-circular, and $H / V$ polarization. We found that $\left\langle P_{s}^{(6)}\right\rangle \geq 0.922 \pm 0.055$, indicating that it is legitimate to use PI tomography, which for six qubits only requires 25 more settings [16]. Under the same experimental conditions as before and 4 min of data collection per setting, we performed the experiment within 2 h only. The density matrix $\varrho_{\mathrm{PI}}$ obtained is shown in Fig. 2(b), with its symmetric subspace shown in Fig. 3(a). The fidelities with the symmetric Dicke states for PI tomography can be found again in Table I. (For the projector to the Dicke state $\left|D_{N}^{(n)}\right\rangle$, all $\left\{O_{j}\right\}_{k l}=0$, except for $\left\{O_{N / 2}\right\}_{n+1, n+1}=1$.) The overlap between the
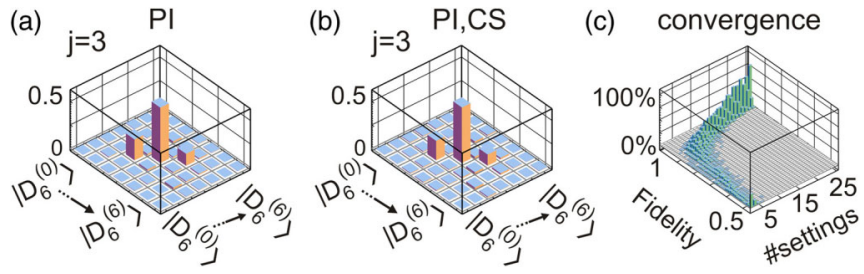

FIG. 3 (color online). Symmetric subspaces $(j=3)$ obtained with (a) PI tomography and (b) CS in the PI subspace with 16 settings. The central bars can be associated with the target state $\left|D_{6}^{(3)}\right\rangle$ and the small bars next to it with $\left|D_{6}^{(2)}\right\rangle$ and $\left|D_{6}^{(4)}\right\rangle$ originating from higher-order noise. (c) Probability to observe a certain fidelity for arbitrarily chosen tomographically incomplete sets of settings in comparison with PI tomography from 28 settings. For 16 settings, the overlap is $\geq 0.950$ on average. 
reconstructed states using either full or PI tomography is 0.922 , which is equivalent to the fidelity of 0.923 between full tomography and its PI part. Clearly, PI tomography rapidly and precisely determines the PI component of the state.

PI tomography with CS.-To speed up analysis even further, based on subsets of the data used for PI tomography, we derived the density matrix $\varrho_{\mathrm{PI}, \mathrm{CS}}$; see Fig. 3(b). Here, the fidelity averaged over a series of different samples is above 0.950 for 16 or more settings [Fig. 3(c)]. Again, both methods are compatible within 1 standard deviation. In summary, our results prove that PI tomography (with CS) enables precise state reconstruction with minimal experimental and computational effort.

Application to noise analysis.-As the count rates for six-photon states depend on the cube of the pump power, full QST is not possible for lower pump power within reasonable time and thus does not allow us to analyze the features of multiphoton states obtained from SPDC. As SPDC is a spontaneous process, with certain probability, there are cases where eight photons have been emitted but only six have been detected, leading to an admixture of $\varrho_{D_{6}^{(2)}}$ and $\varrho_{D_{6}^{(4)}}$. Ideally, the amplitude of the two admixtures should be the same, but, due to polarization dependent coupling efficiencies of $H$ and $V$ photons [26,27], this is not the case. Therefore, we extended the noise model [28] to better specify the experimental state using $\varrho_{\mathrm{exp}}^{\text {noise }}(q, \lambda)=(1-q) \varrho_{D_{6}^{(3)}}+q \varrho_{6}^{\text {asym }}(\lambda), \quad$ with $\quad \varrho_{6}^{\text {asym }}(\lambda)=$ $\frac{4}{7} \varrho_{D_{6}^{(3)}}+3 / 14\left[(1+\lambda) \varrho_{D_{6}^{(2)}}+(1-\lambda) \varrho_{D_{6}^{(4)}}\right]$, the noise $q$, and the asymmetry parameter $\lambda$. Both $q$ and $\lambda$ can be determined from the fidelities to the Dicke states (see also the Supplemental Material [16]). At 8.4 W, noise parameters of $q=0.807 \pm 0.013$ and $\lambda=0.234 \pm 0.015$ were obtained from full tomography, which agree well with those from PI tomography $(q=0.867 \pm 0.041$ and $\lambda=0.273 \pm 0.059$ ). After convincing ourselves that (CS) PI tomography is in excellent agreement with full QST, we can now also perform tomography for low pump powers.
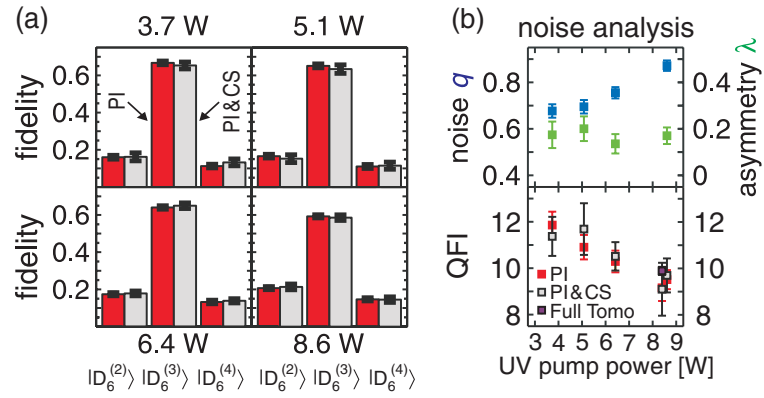

FIG. 4 (color online). (a) Observed fidelities with the states $\left|D_{6}^{(2)}\right\rangle,\left|D_{6}^{(3)}\right\rangle$, and $\left|D_{6}^{(4)}\right\rangle$ at different ultraviolet (UV) pump powers for PI tomography and CS in the PI subspace from 12 settings. The error bars were determined by nonparametric bootstrapping [23]. (b) The influence of the pump power on the higherorder noise expressed via the noise $q$ and the asymmetry parameter $\lambda$ (upper part) and the phase estimation sensitivity expressed via the quantum Fisher information (QFI) (lower part).
We performed PI analysis at 3.7, 5.1, 6.4, and $8.6 \mathrm{~W}$ [see Fig. 4(a)] with sampling times of $67,32,18$, and $15 \mathrm{~h}$ and average counts per setting of 340,390, 510, and 610, respectively. PI tomography shows an increase of the noise parameter $q$ from $0.677 \pm 0.029$ for $3.7 \mathrm{~W}$ to $0.872 \pm 0.023$ for $8.6 \mathrm{~W}$ due to the increasing probability of eight-photon emission for high pump power [29]. Note that the ratio between six-photon detection from eight-photon emission relative to detection from six-photon emission is given by $q /(1-q)$; i.e., for a pump power of $8.6 \mathrm{~W}$, we obtain sixfold detection events with $90 \%$ probability from eight photon emissions, of which two photons were lost. Although fluctuating, the asymmetry parameter $\lambda$ does not show significant dependence on the pump power and lies in the interval $[0.136 \pm 0.042,0.200 \pm 0.053]$ for PI tomography (within $[0.101 \pm 0.116,0.190 \pm 0.071]$ for CS in the PI subspace). This confirms that the difference in the coupling efficiency of $H$ and $V$ does not change with the pump power [see Fig. 4(b)]. The fidelity between the ML fits and the noise model $\varrho_{\exp }^{\text {noise }}(p, \lambda)$ is $>0.925$ for all pump levels, and, for CS in the PI subspace, it is $>0.897$. The high values indicate that our noise model adequately describes the experimental results.

As an example where full knowledge of $\varrho$ is necessary, let us consider the quantum Fisher information $F_{Q}$ which measures the suitability of $\varrho$ to estimate the phase $\theta$ in an evolution $U(\theta, \mathscr{H})=e^{-i \theta \mathscr{H}}[30]$. Here, we want to test whether, in spite of the higher-order noise, the reconstructed states still exhibit sub-shot-noise phase sensitivity. For $\mathscr{H}$, we choose the collective spin operator $J_{x}=1 / 2 \sum_{i=1}^{N} \sigma_{x}^{(i)}$, where $\sigma_{x}^{(i)}$ is $\sigma_{x}$ acting on the $i$ th particle. In the case of $N=6$, a value $F_{Q}>6$ indicates sub-shot-noise phase sensitivity. We observed $11.858 \pm 0.576,10.904 \pm 0.528,10.289 \pm 0.468$, and $9.507 \pm 0.411$ for the corresponding pump powers from 3.7 to $8.6 \mathrm{~W}$ [29] [see Fig. 4(b)]; i.e., sub-shot-noise phase sensitivity is maintained for high pump powers.

Conclusions.-We compared standard quantum state tomography with the significantly more efficient permutationally invariant tomography and also with compressed sensing in the permutationally invariant subspace. For this purpose, we used data of the symmetric Dicke state $\left|D_{6}^{(3)}\right\rangle$ obtained from spontaneous parametric down-conversion of very high pump power. All methods give compatible results within their statistical errors. The number of measurement settings was gradually reduced from 729 for full tomography, to 270 for compressed sensing, to 28 for permutationally invariant tomography, and to only 16 for compressed sensing in the permutationally invariant subspace, giving, in total, a reduction of about a factor of 50 without significantly changing the quantities specifying the state. We applied this highly efficient state reconstruction scheme to study the dependence of higherorder noise on the pump power, clearly demonstrating its benefits for the analysis of multiqubit states required for future quantum computation and quantum simulation applications.

We thank R. Krischek, W. Wieczorek, Z. Zimborás, S. Neuhaus, and L. Knips for stimulating discussions. We 
acknowledge the support of this work by the EU (QWAD, ERC StG GEDENTQOPT, ERC QOLAPS, CHIST-ERA QUASAR, Marie Curie CIG 293993/ENFOQI), the Excellence Initiative of the German Federal and State Governments (ZUK 43), the DFG, FQXi Fund (Silicon Valley Community Foundation), the MINECO (Project No. FIS2012-36673-C03-03), the Basque Government (Project No. IT4720-10), and the National Research Fund of Hungary OTKA (Contract No. K83858). D. G. acknowledges Grants No. W911NF-14-1-0098 and No. W911NF14-1-0133 from the ARO. C. S. thanks QCCC of the Elite Network of Bavaria for support.

[1] H. Häffner, W. Hänsel, C. F. Roos, J. Benhelm, D. Chek-alkar, M. Chwalla, T. Körber, U. D. Rapol, M. Riebe, P. O. Schmidt, C. Becher, O. Gühne, W. Dür and, and R. Blatt, Nature (London) 438, 643 (2005); T. Monz, P. Schindler, J. T. Barreiro, M. Chwalla, D. Nigg, W. A. Coish, M. Harlander, W. Hänsel, M. Hennrich, and R. Blatt, Phys. Rev. Lett. 106, 130506 (2011).

[2] Y.-F. Huang, B.-H. Liu, L. Peng, Y.-H. Li, L. Li, C.-F. Li, and G.-C. Guo, Nat. Commun. 2, 546 (2011); X.-C. Yao, T.-X. Wang, P. Xu, H. Lu, G.-S. Pan, X.-H. Bao, C.-Z. Peng, C.-Y. Lu, Y.-A. Chen, and J.-W. Pan, Nat. Photonics 6, 225 (2012).

[3] D. Gross, Y.-K. Liu, S. T. Flammia, S. Becker, and J. Eisert, Phys. Rev. Lett. 105, 150401 (2010); S. T. Flammia, D. Gross, Y.-K. Liu, and J. Eisert, New J. Phys. 14, 095022 (2012); M. Ohliger, V. Nesme, D. Gross, Y.-K. Liu, and J. Eisert, arXiv:1111.0853; M. Guţă, T. Kypraios, and I. Dryden, New J. Phys. 14, 105002 (2012); M. Ohliger, V. Nesme, and J. Eisert, New J. Phys. 15, 015024 (2013); A. Smith, C. A. Riofrío, B. E. Anderson, H. Sosa-Martinez, I. H. Deutsch, and P. S. Jessen, Phys. Rev. A 87, 030102(R) (2013).

[4] M. Cramer, M. B. Plenio, S. T. Flammia, R. Somma, D. Gross, S. D. Bartlett, O. Landon-Cardinal, D. Poulin, and Y.-K. Liu, Nat. Commun. 1, 149 (2010); O. LandonCardinal and D. Poulin, New J. Phys. 14, 085004 (2012).

[5] T. Baumgratz, D. Gross, M. Cramer, and M. B. Plenio, Phys. Rev. Lett. 111, 020401 (2013); T. Baumgratz, A. Nüßeler, M. Cramer, and M. B. Plenio, New J. Phys. 15, 125004 (2013).

[6] J. O. S. Yin and S. J. van Enk, Phys. Rev. A 83, 062110 (2011).

[7] G. Tóth, W. Wieczorek, D. Gross, R. Krischek, C. Schwemmer, and H. Weinfurter, Phys. Rev. Lett. 105, 250403 (2010).

[8] T. Moroder, P. Hyllus, G. Tóth, C. Schwemmer, A. Niggebaum, S. Gaile, O. Gühne, and H. Weinfurter, New J. Phys. 14, 105001 (2012).

[9] M. Paris and J. Řeháček, Quantum State Estimation (Springer-Verlag, Berlin, Heidelberg, 2004).

[10] N. Kiesel, Ph. D. thesis, Ludwig-Maximilians-Universität München, 2007; D. F. V. James, P. G. Kwiat, W. J. Munro, and A. G. White, Phys. Rev. A 64, 052312 (2001).

[11] A. B. Klimov, G. Björk, and L. L. Sánchez-Soto, Phys. Rev. A 87, 012109 (2013).

[12] For six qubits, we have $P_{s}^{(6)} \geq 2 / 225\left(J_{x}^{2}+J_{y}^{2}+J_{z}^{2}\right)-$ $1 / 90\left(J_{x}^{4}+J_{y}^{4}+J_{z}^{4}\right)+1 / 450\left(J_{x}^{6}+J_{y}^{6}+J_{z}^{6}\right)$ with $J_{i}=$ $\frac{1}{2} \sum_{k} \sigma_{i}^{(k)}$ and $\sigma_{i}^{(k)}$ the application of $\sigma_{i}$ on the $k$ th qubit.

[13] R. B. A. Adamson, P. S. Turner, M. W. Mitchell, and A. M. Steinberg, Phys. Rev. A 78, 033832 (2008).
[14] J. I. Cirac, A. K. Ekert, and C. Macchiavello, Phys. Rev. Lett. 82, 4344 (1999).

[15] Z. Hradil, Phys. Rev. A 55, R1561 (1997).

[16] See Supplemental Material at http://link.aps.org/ supplemental/10.1103/PhysRevLett.113.040503 for additional derivations, which includes Refs. [17,18].

[17] G. Tóth, W. Wieczorek, R. Krischek, N. Kiesel, P. Michelberger, and H. Weinfurter, New J. Phys. 11, 083002 (2009).

[18] For efficient entanglement detection methods for PI density matrices, see L. Novo, T. Moroder, and O. Gühne, Phys. Rev. A 88, 012305 (2013); M. Bergmann and O. Gühne, J. Phys. A 46, 385304 (2013).

[19] S. Boyd and S. Vandenberghe, Convex Optimization (Cambridge University Press, Cambridge, England, 2004).

[20] In order to apply CS and PI tomography together, it suffices that the $\rho_{j}$ matrices in Eq. (1) are of low rank. This also covers the case of global density matrices of comparatively high rank, such as the PI multiqubit singlet state discussed by I. Urizar-Lanz, P. Hyllus, I. L. Egusquiza, M. W. Mitchell, and G. Toth, Phys. Rev. A 88, 013626 (2013). For this highly mixed state, for even $\boldsymbol{N}, \boldsymbol{\rho}_{0}=1, \boldsymbol{p}_{0}=1$, and all other $\boldsymbol{p}_{\boldsymbol{j}}$ 's are 0 .

[21] W. Wieczorek, R. Krischek, N. Kiesel, P. Michelberger, G. Tóth, and H. Weinfurter, Phys. Rev. Lett. 103, 020504 (2009); R. Prevedel, G. Cronenberg, M. S. Tame, M. Paternostro, P. Walther, M. S. Kim, and A. Zeilinger, Phys. Rev. Lett. 103, 020503 (2009).

[22] R. Krischek, W. Wieczorek, A. Ozawa, N. Kiesel, P. Michelberger, T. Udem, and H. Weinfurter, Nat. Photonics 4, 170 (2010).

[23] B. Efron and R. J. Tibshirani, An Introduction to the Bootstrap (Chapman and Hall, London, 1994). The error bars computed via this commonly employed method are a mere quantitative estimate about the fluctuations of the reconstructed state. One should keep in mind that the state reconstruction unavoidably induces systematic errors; see C. Schwemmer, L. Knips, D. Richart, T. Moroder, M. Kleinmann, O. Gühne, and H. Weinfurter, arXiv:1310.8465, which are not recognizable with bootstrap analysis.

[24] The overall runtime of the experiment was almost two weeks, from which $50 \mathrm{~h}$ of useful data could be extracted.

[25] We use the Uhlmann fidelity $F\left(\varrho_{1}, \varrho_{2}\right)=\operatorname{Tr}\left(\sqrt{\sqrt{\varrho_{1}} \varrho_{2} \sqrt{\varrho_{1}}}\right)^{2}$ between the states $\varrho_{1}$ and $\varrho_{2}$ which simplifies to $F\left(\varrho_{1}, Q_{2}\right)=$ $\operatorname{Tr}\left(\varrho_{1} \varrho_{2}\right)$ if one of the two states is pure.

[26] R. S. Bennink, Y. Liu, D. D. Earl, and W. P. Grice, Phys. Rev. A 74, 023802 (2006).

[27] P. Trojek, Ph.D. thesis, Ludwig-Maximilians-Universität München, 2007.

[28] W. Wieczorek, Ph.D. thesis, Ludwig-MaximiliansUniversität München, 2009.

[29] The corresponding noise parameter $q$ determined from CS in the PI subspace with 12 randomly chosen settings is $0.725 \pm 0.052$ for $3.7 \mathrm{~W}$ and $0.886 \pm 0.040$ for $8.6 \mathrm{~W}$. The expectation values for the Fisher information are $11.371 \pm 0.842, \quad 11.685 \pm 1.113, \quad 10.500 \pm 0.613, \quad$ and $9.688 \pm 0.733$ for CS in the PI subspace.

[30] The quantum Fisher information is defined as $F_{Q}(\theta, \mathscr{H})=$ $2 \sum_{i, j}\left(\lambda_{i}-\lambda_{j}\right)^{2} /\left(\lambda_{i}+\lambda_{j}\right)|\langle i|\mathscr{H}| j\rangle|^{2}$ with $\left\{\lambda_{i},|i\rangle\right\}$ the eigenspectrum of $\varrho$; see P. Hyllus, W. Laskowski, R. Krischek, C. Schwemmer, W. Wieczorek, H. Weinfurter, L. Pezzé, and A. Smerzi, Phys. Rev. A 85, 022321 (2012). 